\title{
The Role of MicroRNAs in Influencing Body Growth and Development
}

\author{
Francesca Cirillo Cecilia Catellani Pietro Lazzeroni Chiara Sartori \\ Maria Elisabeth Street \\ Department of Mother and Child, Azienda USL - IRCCS di Reggio Emilia, Reggio Emilia, Italy
}

\section{Keywords}

Growth · Development · MicroRNA · Growth hormone · Insulin-like growth factor-I - Body size

\begin{abstract}
Body growth and development are regulated among others by genetic and epigenetic factors. MicroRNAs (miRNAs) are epigenetic regulators of gene expression that act at the post-transcriptional level, thereby exerting a strong influence on regulatory gene networks. Increasing studies suggest the importance of miRNAs in the regulation of the growth plate and growth hormone (GH)-insulin-like growth factor (IGF) axis during the life course in a broad spectrum of animal species, contributing to longitudinal growth. This review summarizes the role of miRNAs in regulating growth in different in vitro and in vivo models acting on $\mathrm{GH}, \mathrm{GH}$ receptor (GHR), IGFs, and IGF1R genes besides current knowledge in humans, and highlights that this regulatory system is of importance for growth.

๑) 2020 S. Karger AG, Basel
\end{abstract}

\section{Longitudinal Growth and Epigenetics}

Human growth is a complex process regulated by an interplay of different factors comprising a genetic background, the endocrine system, environmental condi- (c) 2020 S. Karger AG, Basel

karger@karger.com

www.karger.com/hrp tions, and more recently epigenetic regulation of gene expression. The individual genetic background plays a pivotal role: studies based on genome-wide association technology have identified more than 400 genes that influence stature and it is possible to estimate that $70-90 \%$ of final height is genetically determined [1-3].

Pharmacogenetic studies show how genetic variants seem to act as a network to drive the different phases of human growth (infancy, childhood, puberty). Moreover, many genetic growth pathways have been shown to be highly evolutionarily conserved (e.g., NOTCH signaling, VEGF signaling, WNT signaling, TGF-beta signaling, and glucocorticoid signaling) [4].

Genetic variants, however, have a small effect size and the variants identified so far can explain no more than $16 \%$ of height variation $[1,3]$. Experimental models and advances in technology have allowed further understanding of growth regulation, in particular within the field of epigenetics, and may contribute to unravel the complexity of the interactions among the abovementioned factors.

Epigenetics has been defined as "the inheritance of variation (-genetics) above and beyond (epi-) changes in the DNA sequence" [5]. There are three main epigenetic mechanisms: DNA methylation, histone modifications, and RNA-based mechanisms.

In this review we will summarize the current knowledge on the role of microRNAs (miRNAs), one of the 
RNA-based epigenetic mechanisms, in the regulation of growth focusing on the regulatory effects of miRNAs on growth hormones $(\mathrm{GH})$, insulin-like growth factors (IGF), and their related receptors. MiRNAs have been shown to contribute to the physiology of growth processes both regulating the hypothalamus-pituitary-IGF axis and regulating growth plate function.

\section{MiRNAs, Biogenesis, and Function}

MiRNAs are short RNAs approximately 22-24 nucleotides in length, which can regulate gene expression at the post-transcriptional level, and represent approximately $30 \%$ of the entire mammalian genome [6,7]. MiRNAs are usually clustered within the human genome both in intergenic regions and intra-genic regions and are transcribed in polycistronic transcripts. MiRNA expression differs depending on the different tissues or organs. Changes in miRNA levels may contribute to explaining some of the variability among phenotypes and can underlie pathological conditions $[8,9]$. In the light of this, therapeutic strategies have been investigated to reinstate physiological miRNA levels. Furthermore, miRNAs are considered to become prognostic and diagnostic molecular markers, because of their stability and easiness to measure [6].

\section{Biogenesis and Action of miRNAs}

At the nuclear level, miRNA genes are transcribed by RNA polymerase II into long primary transcripts (primiRNA) bent in hairpins [10]. Subsequently, Dgcr8 protein, belonging to the RNAse III family, anchors the primiRNA and then Drosha, which also belongs to the RNAse III family, and cleaves the pri-miRNA generating a shorter double stranded pre-miRNA [11].

The pre-miRNA translocates into the cytosol via the XPO5:RAN.GTP complex, where it is bound by Dicer, a cytoplasmic RNAse III complexed with transactivation response element RNA-binding protein (TRBP) [11]. The Dicer:TRBP complex converts the pre-miRNA into a miRNA duplex of about 21-24 nucleotides [12].

The miRNA duplex is loaded in the RNA-induced silencing complex which selects the single-stranded miRNA. The selected mature miRNA, about 22 nucleotides long, contains a seed region which recognizes the mRNA target usually at the $3^{\prime}$ untranslated regions (UTR) but also at the $5^{\prime}$ UTR and at the coding DNA sequence regions [13].

Upon binding, miRNAs determine the repression of translation or degradation of their target mRNAs [14].
Single miRNAs can target several genes and a single gene can be targeted by different miRNAs, revealing a complex interaction network $[15,16]$.

To date, among the 2,300 human mature miRNAs identified, 1,115 are included in miRBase [17]. About 8,500 articles have reported 4,000 miRNAs and 23,000 target genes. Furthermore, about 420,000 miRNA-target interactions are currently recorded in miRTarBase [18]. The biogenesis of miRNAs is summarized in Figure 1.

\section{MiRNA Variation from Birth to Adulthood in Humans}

MiRNA expression changes during the life course because of its importance for the regulation of all biological processes. A recent study in newborns and children showed different miRNA expression levels dependent on different developmental stages [19]. In particular, 36 miRNAs increased from birth to mid-childhood. These miRNAs targeted genes involved in pathways associated with post-translational modifications, metabolism, and immunity. Lai et al. [20] showed that about one third of the miRNAs they analyzed did not show any changes from birth to adulthood, another third was differentially expressed from infancy and adulthood, and the remaining third was detectable only during childhood. This pattern of expression suggested a role of miRNAs in the regulation of genes involved in children's growth and development. It is possible to hypothesize that age-related miRNAs could be involved in biological pathways related with growth and development. This is also suggested by our unpublished data (Fig. 2) from the control subjects of a previous study [21], showing that changes in specific miRNAs regulating insulin-sensitivity occur from childhood to puberty and then from puberty to early adulthood, matching the well-known changes in insulin sensitivity associated with puberty [22].

These concepts are further supported by a study in adulthood showing that age-related miRNAs are related to immune response and regulation of cellular metabolism [23], revealing that miRNAs regulate genes involved in age-related biological mechanisms. Furthermore, miRNA expression has been shown to change significantly in body tissues with age, and different miRNA profiles have been related with longevity in a broad spectrum of animal species, from Caenorhabditis elegans to humans [24-26], as reported in depth below.

More recently, sex-specific temporal patterns of $\mathrm{GH}$ secretion have been demonstrated to regulate miRNA expression levels which in turn regulate sex-differential 


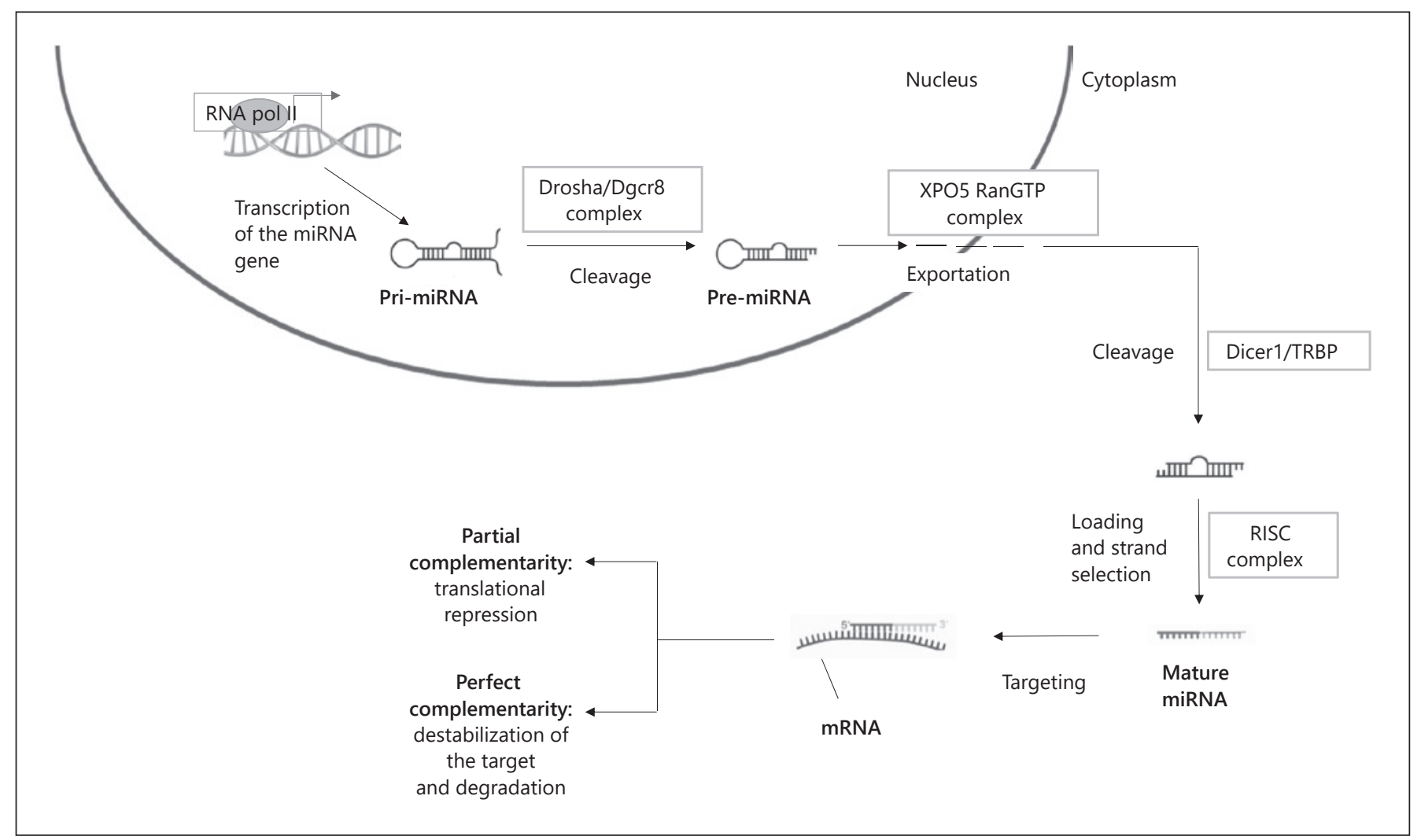

Fig. 1. Biogenesis and action of miRNAs.

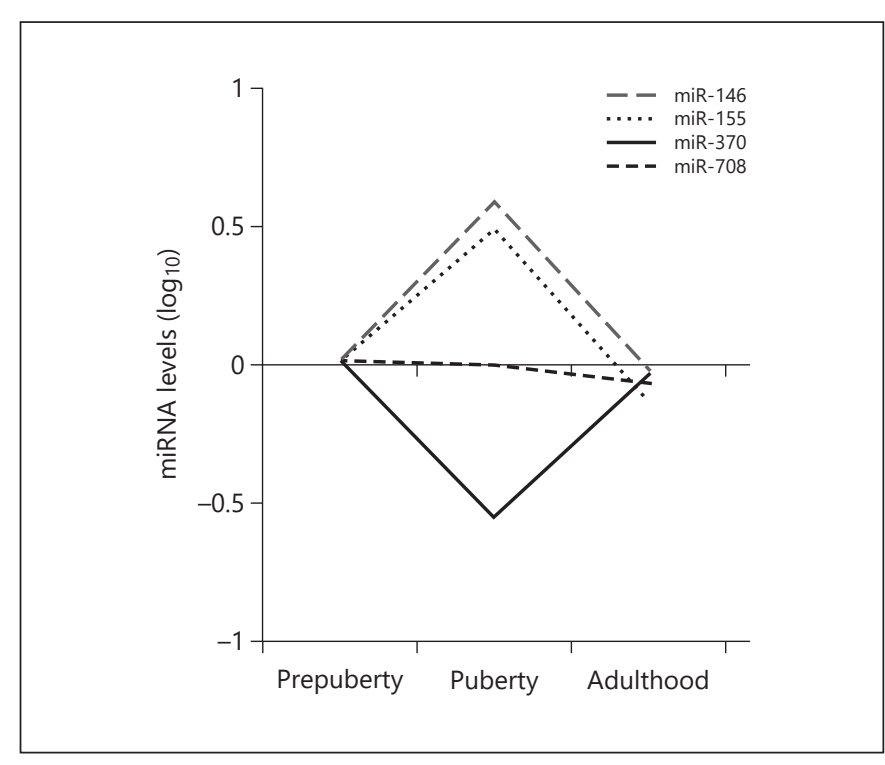

Fig. 2. Changes in specific miRNAs regulating insulin sensitivity from childhood to puberty, and from puberty to early adulthood in healthy subjects $(n=50)$. These subjects are the controls of a previous study, and these data were preliminary to the published data [21].

MiRNAs in Body Growth and Development gene expression. In particular, in the mouse liver, miR1948 is a male-biased miRNA, which represses femalebiased mRNAs, while miR-802 is a female-biased miRNA that represses male-biased mRNAs. At puberty, both of these acquire sex specificity and are activated by the STAT5 transcription factor which belongs to the GH signaling cascade [27]. Therefore, further research should focus on $\mathrm{GH}$-induced gender effects of miRNA regulation. Some findings have suggested that body growth is self-limited by an evolutionarily conserved genetic program in which miRNAs play a pivotal role [28].

\section{MiRNAs Regulating the Growth Plate}

The most important mechanism involved in the growth of the skeleton is endochondral ossification. This process is responsible for the growth of long bones, skull base bones, and for vertebral formation involving a cartilaginous intermediate. Endochondral bone formation occurs in the growth plate, which is a thin layer of cartilage located in the metaphysis of long bones and on the surface 
of plates facing the vertebral bodies. The growth plate shows a high degree of spatial regulation and is histologically formed by three distinct zones: the resting zone, which contains progenitor chondrocytes; the proliferative zone, characterized by chondrocytes that proliferate unidirectionally to form columnar cell clones and produce specific extracellular matrix proteins (e.g., type II collagen and aggrecan), and the hypertrophic zone, in which mature chondrocytes exit the cell cycle, go through hypertrophic differentiation, and express type X collagen. The cartilage matrix then becomes mineralized and terminally differentiated chondrocytes undergo apoptosis. The molecular mechanisms regulating this process are complex and involve different elements: transcription factors, mainly within the Sox family, such as SOX 5, SOX 6 , SOX 9; soluble signaling mediators, with the most important ones being Indian hedgehog (Ihh), parathyroid hormone-related peptide (PTHrP), C natriuretic peptide (CNP), and fibroblast growth factor family members, and extracellular matrix molecules [29-31].

A recent review documented the molecular mechanisms by which miRNAs exert their regulatory role in longitudinal bone growth, and are involved with the regulation of cell growth, particularly of chondrocytes [28]. Two further extensive reviews have evidenced the importance of miRNAs in the regulation of skeletal development, summarizing current knowledge on the role of miRNAs at different bone developmental stages [32] and in different bone cell types [33]. Intriguingly, for the first time the deletion of Dicer, a key enzyme for miRNA biogenesis (see the section entitled Biogenesis and Action of miRNAs; Fig. 1), in murine cartilage evidenced the essential role of miRNAs in controlling chondrocyte proliferation and differentiation [34]. In particular, Dicer-null mice presented severe skeletal growth defects and premature death. Furthermore, the histological cartilage sections from these mice showed a reduction in proliferative chondrocytes and an expansion of the hypertrophic region. Subsequently, specific miRNAs (miR-140 and the let-7 family) were identified to play a key role in this process. Specifically, miR-140 null mice showed an acceleration of hypertrophic differentiation of chondrocytes with advancing endochondral bone formation as a consequence. These mice showed a phenotype characterized by growth retardation and craniofacial deformities [35]. Conversely, the suppression of let- 7 in a mouse model reduced growth plate chondrocyte proliferation and increased cell death, causing mild growth impairment [36]. Interestingly, the mice with both miR-140 deficiency and let-7 downregulation presented severe growth deficiency, similarly to Dicer-null mice, suggesting a pivotal role for the abovementioned miRNAs among others in growth plate development $[32,33,36]$.

More recently, a miRNA profiling study on micro-dissected individual growth plate zones in rats showed a differential expression of 34 miRNAs between the proliferative and the hypertrophic zones [29]. The authors suggested that this expression pattern of miRNAs may be involved in the control of proliferative and differentiative mechanisms which regulate the cell fate of the specific growth plate zones. Moreover, the authors demonstrated that these distinct patterns in growth plate were influenced by the PTHrP concentration gradient across the zones. These findings definitely suggested that the mechanism of action of PTHrP in the control of the growth plate cell function was mediated, at least in part, by miRNAs [29].

Similarly, another recent study showed how rat chondrocytes from the resting zone produced different microvesicles with specific miRNA contents compared to chondrocytes from the proliferative zone. Functional analysis showed that these miRNAs influenced key functions for cartilage development and extracellular matrix control such as stem cell regulation, focal adhesion, and cell cycle control. These data further support the evidence that extracellular miRNAs play a key role for cell-cell communication and cell proliferation and differentiation in the growth plate [37].

\section{MiRNAs Regulating the Hypothalamus-Pituitary and GH/IGF-I Axes}

$\mathrm{GH}$ is the main endocrine regulator of longitudinal growth in humans. GH is a peptide produced by somatotropic cells in the pituitary gland and it exerts its effect promoting growth plate chondrogenesis both directly and via the action of IGF-I, which is its principal mediator $[38,39]$.

MiRNAs are critical for the regulation of hypothalamus function and pituitary development. As GH is produced and released by the anterior pituitary, studies in animal models have focused on this endocrine gland to investigate the role of miRNAs in regulating $\mathrm{GH}$ production and secretion. In conditionally Pitx2-Cre mice knocked-out for the Dicer1 gene, mature miRNAs were found to be decreased in the anterior pituitary, and growth retardation was subsequently observed. This evidenced the essential role of miRNAs in the development of the pituitary gland. The global miRNA profiling per- 
formed in the anterior pituitary cell lines from these mutant mice evidenced specific miRNAs involved in the development of the pituitary gland. Among these, miR-26b was one of the most expressed and in GH3 (somatotrope) pituitary cells, was described to target another miRNA, Lef- 1 , leading to an upregulation of both Pit-1, which is a transcription factor involved in $\mathrm{GH}$ synthesis, and $\mathrm{GH}$ gene expression itself [40]. Interestingly, miR-26b was also described in Yanbian cattle to target EphA2 mRNA, which encodes for a protein belonging to the Receptor tyrosine kinases family which is involved in bone remodeling [41, 42].

More recently, miRNA expression profiles were analyzed in the rat pituitary from postnatal development throughout the entire lifespan showing that specific miRNAs are differentially expressed during pituitary development [43]. In addition, there is evidence that GH synthesis and release are also regulated by miRNAs in mammals [44], as documented below.

Moving downstream along the axis, IGF-I is a crucial hormone for human growth. IGF-I is mainly produced by the liver and its synthesis and secretion are regulated by $\mathrm{GH}$ via $\mathrm{GH}$ receptor (GHR) signaling transduction. IGF-I production has been shown in peripheral tissues, such as bone and cartilage. In target cells, IGF-I controls proliferation, differentiation, and apoptosis. IGF-I bioactivity is regulated by specific binding proteins (IGFBPs) [45]. Although data in the literature concerning the regulation of IGFBPs by miRNAs are present [46], specific data relative to longitudinal growth/body size regulation, miRNAs, and IGFBPs are lacking, and are currently only speculative and thus are not addressed further within this review.

In a recent publication, our group highlighted the links between miRNAs and IGF-I secretion and signaling in chronic inflammation with growth impairment in humans. Bioinformatic analysis regarding miRNAs altered in these conditions revealed a complex network in which miRNAs clearly played a key role in the control of the GHR and IGF-I interactomes [9].

\section{MiRNAs Targeting GH, GHR, IGFs, and IGF1R Regulate Body Growth}

Many studies in the literature have reported the effects of miRNAs on GH and on IGF-I genes in vitro. However, the local and systemic regulation exerted by miRNAs on growth still needs to be further investigated.

In the following sections, the emerging molecular findings concerning the regulatory role of miRNAs on
GH, IGF-I, and their related receptors will be reported. Table 1 summarizes the effects of specific miRNAs on GH, GHR, IGFs, IGF1R, and downstream signaling peptides in different animal species, as reported in the following two sections.

\section{MiRNAs, GH, and GHR}

The role of miRNAs on growth has been studied in several animal models; however, little is known on their role in regulating GH synthesis and secretion. In zebrafish, an important role on embryo growth has been shown relative to the miR-200 family of miRNAs which is composed by six members clustered on chromosomes 6 and 23. In particular, the injection of miR-141 and mir-429a mimics in zebrafish embryos reduced the expression of GH and GHR significantly. Furthermore, miR-141 and mir-429a were shown to be positively regulated in a feedback loop by GH. In detail, an injection of GH in the zebrafish embryos increased the levels of miR-141 and miR$429 \mathrm{a}$ via $\mathrm{p} 53$, and the regulatory action of miR-141 and mir-429a had reflections on somatic growth as the overexpression of miR-141/429a drastically reduced the embryo body length; conversely, the use of the respective miRNA-inhibitors rescued the defective growth [47] (Table 1).

In a recent study of the rat pituitary gland, among the miRNAs profiled and found to be differentially expressed throughout the growth process, 15 miRNAs were involved in GH signaling. Among these, miR-141-3p regulated $\mathrm{GH}$ expression negatively, resulting in a reduction in GH1 mRNA levels. Conversely, the use of an miR-141$3 p$ inhibitor significantly increased $\mathrm{GH} 1$ gene expression [43] (Table 1).

Further data emerged from a study in swine primary anterior pituitary cells cultivated separately with GH-releasing hormone $(\mathrm{GHRH})$, which positively regulates GH secretion, and corticostatin (CST), which has an opposite effect. The high-throughput miRNA microarray analysis that was performed evidenced 19 (12 upregulated and 7 downregulated) and 35 (21 upregulated and 14 downregulated) differentially expressed miRNAs in response to the two treatments, respectively. Among these miRNAs, miR-361-5p and $\mathrm{miR}-127$ were regulated in opposite ways under the two treatments, suggesting a potential role in $\mathrm{GH}$ regulation which still needs further investigation. Interestingly, let-7c, which was upregulated under CST treatment, negatively regulated GH production and release, directly targeting $\mathrm{GH} 1$ and GHRH [44] (Table 1). 
Table 1. Specific effects of miRNAs on GH, GHR, IGFs, IGF1R, and on downstream signaling peptides in different animal species

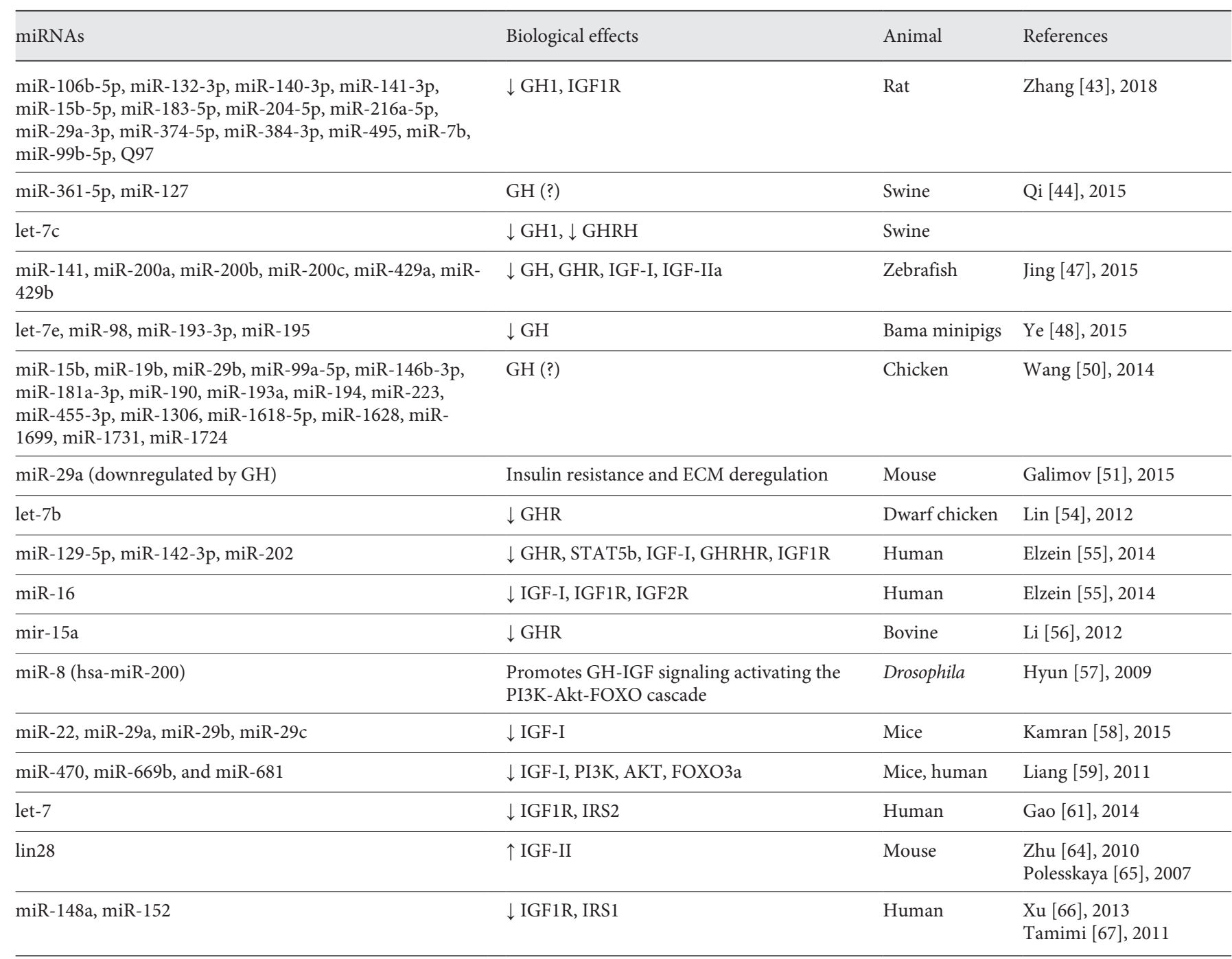

An additional animal study using a miRNA microarray analysis approach was conducted on anterior pituitary from Bama minipigs, a miniature pig breed with a lower growth performance compared to healthy control animals. In this study, 41 miRNAs were shown to be differentially expressed ( 32 upregulated and 9 downregulated), and among the upregulated miRNAs, let7e, miR-98, miR-193-3p, and miR-195 were associated with decreased GH secretion in the pituitary [48] (Table 1).

Recently, a relevant study in mice focused on MIR205HG, which is the host gene for miR-205 that also gives origin to a long noncoding RNA, which regulates the anterior pituitary transcriptome [49]. Interestingly, $\mathrm{GH}$ itself exerts a regulatory action on miRNAs. Firstly, in chicken hepatocytes, it was reported that GH treatment upregulated 16 miRNAs and downregulated 1 miRNA (miR-1724). These miRNAs were all predicted to target $\mathrm{GH}$-regulated mRNAs, involved in both growth and metabolism [50] (Table 1).

In a $\mathrm{GH}$-deficient mouse model, $\mathrm{GH}$ replacement therapy determined a downregulation of miR-29a and this was associated with insulin resistance in skeletal muscle. Specifically, miR-29a targeted genes that are negative regulators of insulin signaling (e.g., PTEN), as well as proinflammatory and profibrotic genes (e.g., FSTL1 and SPARC, respectively) involved in extracellular matrix organization [51] (Table 1).

It is well known that $\mathrm{GH}$ exerts its biological functions by binding to its receptor (GHR) on target cells, 
thus activating multiple intracellular pathways and leading to changes in gene expression, cell differentiation, and metabolism [52]. Furthermore, GHR deficiency is associated with growth and metabolic disorders while, conversely, its increased expression is related with several diseases, such as cancer [53]. Due to its role, GHR expression is tightly controlled by several mechanisms, including miRNAs. In dwarf chicken, let$7 \mathrm{~b}$ directly targets the GHR transcript and growth retardation was observed if a $3^{\prime}$-UTR region GHR gene deletion was created, as this determines the loss of the let-7b target site necessary for the regulation of GHR expression [54] (Table 1).

Studies in cancer human cell lines (MCF7 and LNCaP) and in control cells (HEK293) showed that among the miRNAs predicted to target the $3^{\prime}$-UTR region of the human GHR gene, four miRNAs have been identified to inhibit the GHR gene transcript. Among these, miR-129-5p had putative binding sites also on STAT5b, a downstream effector of both the GH and IGF signaling pathways. In addition, miR-202 was predicted to target GHRHR, IGFI, IGF1R, and cytokine-induced suppressor, and miR-16 to target IGF-I, IGF1R, and IGF2R, also suggesting an important regulatory role for miRNAs at different steps of the GH/IGF-I axis [55] (Table 1). Using miRNA prediction software, miR-15a, which belongs to the same family as miR-16, was predicted to target GHR mRNA and, subsequently, miR-15a was confirmed to inhibit the expression of GHR in bovine mammary epithelial cell lines [56] (Table 1).

\section{MiRNAs, IGF-I, IGF-II, and IGF1R}

The most relevant findings regarding the regulatory role of miRNAs on IGF-I, IGF-II, and their related receptor, IGF1R, have emerged in the last decade. Studies conducted in larvae of Drosophila melanogaster have shown that miR- 8 activates the PI3K-Akt-FOXO cascade, shared by IGFs and insulin, which promotes the growth of body fat cells [57] (Table 1). Consistently, flies knocked out for miR-8 were smaller in size and had impaired insulin signaling. These data have suggested that the human homolog of miR-8, miR-200, could play an important role in body growth and metabolism, showing a new regulatory function in the insulin/IGF pathway [57].

Emerging data have suggested that miRNAs could have a key role in regulating IGF-I and IGF-II. Experimental analyses conducted in zebrafish revealed that miR-141 and miR-429a, belonging to the highly conserved miR-200 family, were involved in embryo size control, as described above. By using a mimic/inhibitor
miRNA approach, it was demonstrated that both miR141 and miR-429a dramatically reduced the expression of both IGF-I and IGF-IIa by directly targeting several genes involved in the GH/IGF axis [47] (Table 1).

A study conducted in mice at different weeks from birth analyzed the miRNA profiling in kidney and lung tissues. Four miRNAs increased in multiple tissues with age, and, among these, 3 belonging to the miR-29 family (miR-29a, miR-29b, and miR-29c) targeted IGF-I mRNA, among others. Subsequently, in vitro experiments conducted in human embryonic kidney cells overexpressing miR-29a, miR-29b, and miR-29c validated the IGF-I transcript as a direct target of this family of miRNAs [58] (Table 1).

An additional study reported the findings from a miRNA array screening on brain tissue from two mutant long-lived mouse models identifying three miRNAs upregulated in the hippocampus that decreased the downstream effectors of the IGF-I signaling pathway. In detail, $\mathrm{PI} 3 \mathrm{~K}, \mathrm{AKT}$, and FOXO3a protein contents were reduced in brain tissues. These results were further confirmed in in vitro cell lines of human fibroblasts, where miR-470, miR-669b, and miR-681 inhibited IGF-I signaling if overexpressed [59] (Table 1).

A few studies have focused primarily on the IGF1R, and have integrated the concept that miRNAs are epigenetic molecular tools that carry out an important action on the GH/IGF-I axis in the context of growth. Intriguingly, in humans, about $31 \%$ of loci associated with body length have been previously identified as predicted Let-7 targets [60], and among these IGF1R and IRS-2, a downstream effector of the IGF signaling cascade, were validated target genes in vitro [61] (Table 1). An interesting study documented the role of the Let- 7 family of miRNAs in regulating body size [62], and transgenic mouse models expressing Lin28, an inhibitor of Let-7 [63], showed an increase in body size and crown-rump length [64] (Table 1).

Lin28 was reported to control the expression of IGF-II functioning as a "translational enhancer" in in vitro murine myoblasts [65] (Table 1). As reported in the previous section [43], 15 miRNAs in the rat were shown to regulate the IGF1R gene. Finally, further information concerning the regulation of IGF1R as well as IRS1, a downstream adaptor molecule of the IGF signaling cascade, was provided by an in vitro study conducted in both human breast cancer cell lines and tissues, proving that both were novel target genes for miR-148a and miR-152 [66, 67] (Table 1). 


\section{Conclusions}

Overall, these findings highlight a role for miRNAs in regulating longitudinal growth by regulating processes within the growth plate, and also in the central nervous system and peripherally through effects on GH secretion and signaling, IGF synthesis, and action. Most of the evidence to date comes from animal models and in vitro studies, but is clearly relevant for human growth and disease, and warrants further research.

\section{Statement of Ethics}

The authors have no ethical conflicts to disclose.

\section{Disclosure Statement}

The authors have no conflicts of interest to declare.

\section{Funding Sources}

This research received no external funding.

\section{Author Contributions}

Conceptualization, M.E.S. Writing - original draft preparation, F.C., C.C., P.L., C.S. Visualization, F.C., C.C., M.E.S. Supervision, M.E.S. Writing - review and editing, F.C., C.C., P.L., C.S., M.E.S.

\section{References}

1 Wood AR, Esko T, Yang J, Vedantam S, Pers $\mathrm{TH}$, Gustafsson S, et al.; Electronic Medical Records and Genomics (eMEMERGEGE) Consortium; MIGen Consortium; PAGEGE Consortium; LifeLines Cohort Study. Defining the role of common variation in the genomic and biological architecture of adult human height. Nat Genet. 2014 Nov;46(11):1173-86.

2 Lanktree MB, Guo Y, Murtaza M, Glessner JT, Bailey SD, Onland-Moret NC, et al. Metaanalysis of dense genecentric association studies reveals common and uncommon variants associated with height. Am J Hum Genet. $2011 \mathrm{Jan} ; 88(1): 6-18$

3 Lango Allen H, Estrada K, Lettre G, Berndt SI, Weedon MN, Rivadeneira F, et al. Hundreds of variants clustered in genomic loci and biological pathways affect human height. Nature. 2010 Oct;467(7317):832-8.

4 Stevens A, De Leonibus C, Whatmore A, Hanson D, Murray P, Chatelain P, et al. Pharmacogenomics related to growth disorders. Horm Res Paediatr. 2013;80(6):477-90.

5 Bonasio R, Tu S, Reinberg D. Molecular signals of epigenetic states. Science. 2010 Oct; 330(6004):612-6.

6 Bartel DP. MicroRNAs: target recognition and regulatory functions. Cell. 2009 Jan; 136(2):215-33.

7 Filipowicz W, Bhattacharyya SN, Sonenberg N. Mechanisms of post-transcriptional regulation by microRNAs: are the answers in sight? Nat Rev Genet. 2008 Feb;9(2):102-14.

8 Cirillo F, Catellani C, Sartori C, Lazzeroni P, Amarri S, Street ME. Obesity, insulin resistance, and colorectal cancer: could miRNA dysregulation play a role? Int J Mol Sci. 2019 Jun;20(12):E2922.

9 Cirillo F, Lazzeroni P, Catellani C, Sartori C, Amarri S, Street ME. MicroRNAs link chronic inflammation in childhood to growth impairment and insulin-resistance. Cytokine Growth Factor Rev. 2018 Feb;39:1-18.
10 Denli AM, Tops BB, Plasterk RH, Ketting RF, Hannon GJ. Processing of primary microRNAs by the Microprocessor complex. Nature. 2004 Nov;432(7014):231-5.

11 Kok KH, Ng MH, Ching YP, Jin DY. Human TRBP and PACT directly interact with each other and associate with dicer to facilitate the production of small interfering RNA. J Biol Chem. 2007 Jun;282(24):17649-57.

12 Chendrimada TP, Gregory RI, Kumaraswamy E, Norman J, Cooch N, Nishikura K, et al. TRBP recruits the Dicer complex to Ago2 for microRNA processing and gene silencing. Nature. 2005 Aug;436(7051):740-4.

13 Krützfeldt J, Stoffel M. MicroRNAs: a new class of regulatory genes affecting metabolism. Cell Metab. 2006 Jul;4(1):9-12.

14 Pillai RS, Bhattacharyya SN, Filipowicz W. Repression of protein synthesis by miRNAs: how many mechanisms? Trends Cell Biol. 2007 Mar; 17(3):118-26.

15 Faghihi MA, Modarresi F, Khalil AM, Wood DE, Sahagan BG, Morgan TE, et al. Expression of a noncoding RNA is elevated in Alzheimer's disease and drives rapid feed-forward regulation of beta-secretase. Nat Med. 2008 Jul;14(7):723-30.

16 Timmons JA, Good L. Does everything now make (anti)sense? Biochem Soc Trans. 2006 Dec;34(Pt 6):1148-50.

17 Alles J, Fehlmann T, Fischer U, Backes C, Galata V, Minet M, et al. An estimate of the total number of true human miRNAs. Nucleic Acids Res. 2019 Apr;47(7):3353-64

18 Chou CH, Shrestha S, Yang CD, Chang NW, Lin YL, Liao KW, et al. miRTarBase update 2018: a resource for experimentally validated microRNA-target interactions. Nucleic Acids Res. 2018 Jan;46(D1):D296-302.
19 Huen K, Lizarraga D, Kogut K, Eskenazi B, Holland N. Age-Related Differences in miRNA Expression in Mexican-American Newborns and Children. Int J Environ Res Public Health. 2019 Feb;16(4):E524.

20 Lai CY, Wu YT, Yu SL, Yu YH, Lee SY, Liu $\mathrm{CM}$, et al. Modulated expression of human peripheral blood microRNAs from infancy to adulthood and its role in aging. Aging Cell. 2014 Aug;13(4):679-89.

21 Montanini L, Smerieri A, Gullì M, Cirillo F, Pisi G, Sartori C, et al. miR-146a, miR-155, miR-370, and miR-708 Are CFTR-Dependent, Predicted FOXO1 Regulators and Change at Onset of CFRDs. J Clin Endocrinol Metab. 2016 Dec;101(12):4955-63.

22 Kelsey MM, Zeitler PS. Insulin Resistance of Puberty. Curr Diab Rep. 2016 Jul;16(7):64.

23 Huan T, Chen G, Liu C, Bhattacharya A, Rong $\mathrm{J}$, Chen $\mathrm{BH}$, et al. Age-associated microRNA expression in human peripheral blood is associated with all-cause mortality and age-related traits. Aging Cell. 2018 Feb;17(1):e12687.

24 Lee BP, Burić I, George-Pandeth A, Flurkey $\mathrm{K}$, Harrison DE, Yuan R, et al. MicroRNAs miR-203-3p, miR-664-3p and miR-708-5p are associated with median strain lifespan in mice. Sci Rep. 2017 Mar;7(1):44620.

25 Mimura S, Iwama H, Kato K, Nomura K, Kobayashi M, Yoneyama $\mathrm{H}$, et al. Profile of microRNAs associated with aging in rat liver. Int J Mol Med. 2014 Oct;34(4):1065-72.

26 Noren Hooten N, Fitzpatrick M, Wood WH 3rd, De S, Ejiogu N, Zhang Y, et al. Age-related changes in microRNA levels in serum. Aging (Albany NY). 2013 Oct;5(10):725-40.

27 Hao P, Waxman DJ. Functional Roles of SexBiased, Growth Hormone-Regulated MicroRNAs miR-1948 and miR-802 in Young Adult Mouse Liver. Endocrinology. 2018 Mar;159(3):1377-92.

28 Lui JC. Regulation of body growth by microRNAs. Mol Cell Endocrinol. 2017 Nov;456:2-8. 
29 Jee YH, Wang J, Yue S, Jennings M, Clokie SJ, Nilsson O, et al. mir-374-5p, mir-379-5p, and mir-503-5p Regulate Proliferation and Hypertrophic Differentiation of Growth Plate Chondrocytes in Male Rats. Endocrinology. 2018 Mar;159(3):1469-78.

30 Cirillo F, Lazzeroni P, Sartori C, Street ME Inflammatory Diseases and Growth: Effects on the GH-IGF Axis and on Growth Plate. Int J Mol Sci. 2017 Aug;18(9):E1878.

31 Michigami T. Regulatory mechanisms for the development of growth plate cartilage. Cell Mol Life Sci. 2013 Nov;70(22):4213-21.

32 Papaioannou G. miRNAs in bone development. Curr Genomics. 2015 Dec;16(6):42734. doi: https://doi.org/10.2174/13892029166 66150817202425

33 Papaioannou G, Mirzamohammadi F, Kobayashi T. MicroRNAs involved in bone formation. Cell Mol Life Sci. 2014 Dec;71(24): 4747-61.

34 Kobayashi T, Lu J, Cobb BS, Rodda SJ, McMahon AP, Schipani E, et al. Dicer-dependent pathways regulate chondrocyte proliferation and differentiation. Proc Natl Acad Sci USA. 2008 Feb;105(6):1949-54.

35 Nakamura Y, Inloes JB, Katagiri T, Kobayashi T. Chondrocyte-specific microRNA-140 regulates endochondral bone development and targets Dnpep to modulate bone morphogenetic protein signaling. Mol Cell Biol. 2011 Jul;31(14):3019-28.

36 Papaioannou G, Inloes JB, Nakamura Y, Paltrinieri E, Kobayashi T. let-7 and miR-140 microRNAs coordinately regulate skeletal development. Proc Natl Acad Sci USA. 2013 Aug; 110(35):E3291-300.

37 Lin Z, McClure MJ, Zhao J, Ramey AN, Asmussen N, Hyzy SL, et al. MicroRNA Contents in Matrix Vesicles Produced by Growth Plate Chondrocytes are Cell Maturation Dependent. Sci Rep. 2018 Feb;8(1):3609.

38 Blum WF, Alherbish A, Alsagheir A, El Awwa A, Kaplan W, Koledova E, et al. The growth hormone-insulin-like growth factor-I axis in the diagnosis and treatment of growth disorders. Endocr Connect. 2018 Jun;7(6):R21222.

39 Savage MO, Burren CP, Rosenfeld RG. The continuum of growth hormone-IGF-I axis defects causing short stature: diagnostic and therapeutic challenges. Clin Endocrinol. 2010 Jun;72(6):721-8.

40 Zhang Z, Florez S, Gutierrez-Hartmann A, Martin JF, Amendt BA. MicroRNAs regulate pituitary development, and microRNA $26 \mathrm{~b}$ specifically targets lymphoid enhancer factor 1 (Lef-1), which modulates pituitary transcription factor 1 (Pit-1) expression. J Biol Chem. 2010 Nov;285(45):34718-28.

41 Yuan B, Yu WY, Dai LS, Gao Y, Ding Y, Yu $\mathrm{XF}$, et al. Expression of microRNA-26b and identification of its target gene EphA2 in pituitary tissues in Yanbian cattle. Mol Med Rep. 2015 Oct;12(4):5753-61.
42 Park JE, Son AI, Zhou R. Roles of EphA2 in development and disease. Genes (Basel). 2013 Jul;4(3):334-57.

43 Zhang H, Qi Q, Chen T, Luo J, Xi Q, Jiang Q, et al. Age-related changes in MicroRNA in the rat pituitary and potential role in GH regulation. Int J Mol Sci. 2018 Jul;19(7):E2058.

44 Qi QE, Xi QY, Ye RS, Chen T, Cheng X, Li CY, et al. Alteration of the miRNA expression profile in male porcine anterior pituitary cells in response to GHRH and CST and analysis of the potential roles for miRNAs in regulating GH. Growth Horm IGF Res. 2015 Apr; 25(2):66-74.

45 Bach LA. IGF-binding proteins. J Mol Endocrinol. 2018 Jul;61(1):T11-28.

46 El Tayebi HM, Abdelaziz AI. Epigenetic regulation of insulin-like growth factor axis in hepatocellular carcinoma. World J Gastroenterol. 2016 Mar;22(9):2668-77.

47 Jing J, Xiong S, Li Z, Wu J, Zhou L, Gui JF, et al. A feedback regulatory loop involving p53/ miR-200 and growth hormone endocrine axis controls embryo size of zebrafish. Sci Rep. 2015 Oct;5(1):15906.

48 Ye RS, Li M, Qi QE, Cheng X, Chen T, Li CY, et al. Comparative anterior pituitary miRNA and mRNA expression profiles of bama minipigs and landrace pigs reveal potential molecular network involved in animal postnatal growth. PLoS One. 2015 Jul;10(7):e0131987.

49 Du Q, Hoover AR, Dozmorov I, Raj P, Khan S, Molina E, et al. MIR205HG is a long noncoding RNA that regulates growth hormone and prolactin production in the anterior pituitary. Dev Cell. 2019 May;49(4):618-631.e5.

50 Wang X, Yang L, Wang H, Shao F, Yu J, Jiang $\mathrm{H}$, et al. Growth hormone-regulated mRNAs and miRNAs in chicken hepatocytes. PLoS One. 2014 Nov;9(11):e112896.

51 Galimov A, Hartung A, Trepp R, Mader A, Flück M, Linke A, et al. Growth hormone replacement therapy regulates microRNA-29a and targets involved in insulin resistance. J Mol Med. 2015 Dec;93(12):1369-79.

52 Bergan-Roller HE, Sheridan MA. The growth hormone signaling system: insights into coordinating the anabolic and catabolic actions of growth hormone. Gen Comp Endocrinol. 2018 Mar;258:119-33.

53 Dehkhoda F, Lee CMM, Medina J, Brooks AJ. The growth hormone receptor: mechanism of receptor activation, cell signaling, and physiological aspects. Front Endocrinol. $2018 \mathrm{Feb}$ 13;9:35. doi: https://doi.org/10.3389/fendo. 2018.00035 .

54 Lin S, Li H, Mu H, Luo W, Li Y, Jia X, et al Let- $7 \mathrm{~b}$ regulates the expression of the growth hormone receptor gene in deletion-type dwarf chickens. BMC Genomics. 2012 Jul; 13(1):306.
55 Elzein S, Goodyer CG. Regulation of human growth hormone receptor expression by $\mathrm{mi}$ croRNAs. Mol Endocrinol. 2014 Sep;28(9): 1448-59.

56 Li HM, Wang CM, Li QZ, Gao XJ. MiR-15a decreases bovine mammary epithelial cell viability and lactation and regulates growth hormone receptor expression. Molecules. 2012 Oct; 17(10):12037-48.

57 Hyun S, Lee JH, Jin H, Nam J, Namkoong B, Lee G, et al. Conserved MicroRNA miR-8/ miR-200 and its target USH/FOG2 control growth by regulating PI3K. Cell. 2009 Dec; 139(6):1096-108

58 Kamran F, Andrade AC, Nella AA, Clokie SJ, Rezvani G, Nilsson O, et al. Evidence that upregulation of MicroRNA-29 contributes to postnatal body growth deceleration. Mol Endocrinol. 2015 Jun;29(6):921-32.

59 Liang R, Khanna A, Muthusamy S, Li N, Sarojini $\mathrm{H}$, Kopchick JJ, et al. Post-transcriptional regulation of IGF1R by key microRNAs in long-lived mutant mice. Aging Cell. 2011 Dec;10(6):1080-8.

60 Lettre G, Jackson AU, Gieger C, Schumacher FR, Berndt SI, Sanna S, et al. Identification of ten loci associated with height highlights new biological pathways in human growth. Nat Genet. 2008 May;40(5):584-91.

61 Gao L, Wang X, Wang X, Zhang L, Qiang C, Chang S, et al. IGF-1R, a target of let-7b, mediates crosstalk between IRS-2/Akt and MAPK pathways to promote proliferation of oral squamous cell carcinoma. Oncotarget. 2014 May;5(9):2562-74.

62 Frost RJ, Olson EN. Control of glucose homeostasis and insulin sensitivity by the Let-7 family of microRNAs. Proc Natl Acad Sci USA. 2011 Dec;108(52):21075-80.

63 Ali PS, Ghoshdastider U, Hoffmann J, Brutschy B, Filipek S. Recognition of the let$7 \mathrm{~g}$ miRNA precursor by human Lin28B. FEBS Lett. 2012 Nov;586(22):3986-90.

64 Zhu H, Shah S, Shyh-Chang N, Shinoda G, Einhorn WS, Viswanathan SR, et al. Lin28a transgenic mice manifest size and puberty phenotypes identified in human genetic association studies. Nat Genet. 2010 Jul;42(7): 626-30.

65 Polesskaya A, Cuvellier S, Naguibneva I, Duquet A, Moss EG, Harel-Bellan A. Lin-28 binds IGF-2 mRNA and participates in skeletal myogenesis by increasing translation efficiency. Genes Dev. 2007 May;21(9):112538.

66 Xu Q, Jiang Y, Yin Y, Li Q, He J, Jing Y, et al. A regulatory circuit of miR-148a/152 and DNMT1 in modulating cell transformation and tumor angiogenesis through IGF-IR and IRS1. J Mol Cell Biol. 2013 Feb;5(1):3-13.

67 Tamimi RM, Colditz GA, Wang Y, Collins LC, Hu R, Rosner B, et al. Expression of IGF1R in normal breast tissue and subsequent risk of breast cancer. Breast Cancer Res Treat. 2011 Jul;128(1):243-50. 